\title{
Low-temperature plasma radiofrequency ablation of Sphenopalatine ganglion in Cluster headache: a case report
}

\section{Xiaoping Wang ( $\sim$ bjmuxp@163.com )}

University of Pittsburgh Medical Center Health System Pain Medicine Program

\section{Liqiang Yang}

Pain Medicine, Xuanwu Hospital, Capital Meidical University

\section{Yuna Guo}

Pain Medicine, Xuanwu Hospital, Capital Medical University

\section{Jiaxiang Ni}

Pain Medicine, Xuanwu Hospital, Capital Medical University,

\section{Yuanzhang Tang}

Pain Medicine, Xuanwu Hospital, Capital Medical University

\section{Case report}

Keywords: cluster headache, coblation, herpes zoster

Posted Date: January 2nd, 2020

DOI: https://doi.org/10.21203/rs.2.19826/v1

License: (c) (i) This work is licensed under a Creative Commons Attribution 4.0 International License. Read Full License 


\section{Abstract}

Background $\mathbb{Z}$ Herpes zoster is a common disease mainly affect sensory nerves, but in this report sphenopalatine ganglion (SPG), mainly a parasympathetic ganglion was infected by the vesicular-zoster virus, with the performance of cluster headache.

Case presentation: The patient underwent headache on left side orbit, supraorbital and temporal regions after infection of herpes zoster for 8 months, with lacrimation, conjunctival injection and nasal discharge. Pulsed radiofrequency of the SPG under ultrasound guidance was performed, we applied 2 cycles of PRF at $42^{\circ} \mathrm{C}$ for 120 seconds each time. Partial response lasted 1 month and the pain had recover to its baseline level. Low-temperature plasma radiofrequency ablation (coblation) technology is a relatively new technology that has shown promise in treating neuropathic pain. Then a CT-guided coblation of SPG was performed, used three of intensity for coblation (lasting for 30s, repeat twice) and six of intensity for coagulation (lasting for 30s, once). After coblation, the patient reported completed pain relief and autonomic symptoms disappeared without any side effects. The patient was satisfied with the effect on post-operative telephone follow-up performed on day 7, month 1 and month 3, pain VAS score remained on $0 / 10$.

Conclusions: Our report demonstrates that coblation technology is succeseful treatment for cluster headache in this case, but this finding still needs additional studies for confirmation.

Key words: cluster headache, coblation, herpes zoster

\section{Introduction}

Cluster headache $(\mathrm{CH})$ is a rare but probably the most severe pain known, characterized by ipsilateral headache, lasting 50 to 180 minutes and occurring as many as 8 times per day. with pain localized to the orbit, supraorbital and/or temporal regions and associated autonomic features. Autonomic symptoms, such as lacrimation (tearing), conjunctival injection (redness of the sclera), rhinorrhoea, nasal congestion, hyperhidrosis (excessive sweating) and eyelid oedema, usually occur on the ipsilateral side to the pain[1].

Herpes zoster $(\mathrm{HZ})$ is a common disease results from reactivation of the vesicular-zoster virus(VZV) which persisted asymptomatically in the ganglia of sensory cranial nerves and spinal dorsal root ganglia, and spread along the sensory nerve to the dermatome. With a performance of painful, blistering skin eruption in a dermatomal distribution. Trigeminal nerve is the most frequently involved site for $\mathrm{HZ}[2]$. There was rare report of $\mathrm{CH}$ after $\mathrm{HZ}$, in which the VZV would infect the sphenopalatine ganglion (SPG). Here, we report a case of $\mathrm{CH}$ after $\mathrm{HZ}$, who treated with pulsed radiofrequency and low-temperature plasma radiofrequency ablation (coblation).

\section{Case Report}


A 86-year-old female patient underwent headache on left side 8 months ago, with pain localized to the orbit, supraorbital and temporal regions, and 2 days later skin eruption appeared on the painful area. Then, herpes zoster was diagnosed and pain became severe despite antiviral therapy, pain was described as a continuous stabbing pain with lacrimation, conjunctival injection and nasal discharge. The pain VAS score was $8-9 / 10$ ( 0 is no pain and 10 is the most severe pain). Pain occurs twice a day at morning and 4 p.m and lasting 2 hours.

Pulsed radiofrequency of the sphenopalatine ganglion (SPG) under ultrasound guidance was performed. The patient was placed in the lateral position with the cervical spine in the neutral position. The coronoid notch was identified by asking the patient to open and close the mouth and palpating just caudal to the middle point of zygomatic arch. The skin was prepared with antiseptic solution. A curved transducer was then placed in the transverse plane directly over the mandibular notch and asked the patient to slightly open the mouth. The masseter muscle was easily identified by following its origin on the zygomatic srch. Just below and deep to the masseter muscle was the hyperechoic and parallel lateral pterygoid plate and ventral to it was the vertical maxillary bone, the pterygopalatine fossa was just between these two bony structure (Fig. 1). The ultrasound transducer was moved slightly craniad and cauded to identify the full legth of pterygopalatine fossa and the distribution of vessels beside the fossa under color Doppler (Fig. 2). The in-plane puncture path was designed from the dorsal part of the transducer to the fossa and avoid the injury of blood vessels. A 21 -gauge, $10-\mathrm{cm}$ straight radiofrequency needle with a 5 -mm active tip (Cosman,USA) was inserted to the fossa under real-time ultrasound guidance using an in-plane approach. The position of the needle tip was confirmed in the fossa with lateral fluoroscopic image, which just like a "little Chillies"(Fig. 3). The needle core was then removed and replaced with a radiofrequency electrode (CSK-10,Cosman,USA) connected to a radiofrequency generator to measure sensory threshold using $50 \mathrm{~Hz}$ electrical stimulation. Electrical current of $0.5 \mathrm{~V}$ could induce paresthesia of the nasal root. Then pulsed radiofrequency mode was set with a maximum temperature of $42^{\circ} \mathrm{C}$, patient was treated twice for 120 seconds each time. The pain was relieved significantly after the procedure with VAS score declined to $1-2$ of 10 , but the patient complain the pain had recover 1 month after the procedure with the same severity and area compare to prior treatment.

The patient returned to our department and asked to perform a neurolysis of the ganglion, for afraid of postoperation facial numbness, the coblation of the sphenopalatine ganglion was performed under CT guidance according to our experience in treating trigeminal neuralgia[3], the puncture path was designed used thin-slice CT scans ( $1 \mathrm{~mm} /$ layer), the depth from skin to pterygopalatine fossa was $58.63 \mathrm{~mm}$ (Fig. 4). The Gaotong $17 \mathrm{G}$ puncture trocar was inserted to the fossa, and 3D-CT scan to confirm the needle tip (Fig. 5). Then, a coblation needle (DXR-G1100-A185;Xi'an Gaotong Medical Technology Company,LTD,China), attached to operation system (SM-D380D;Xi'an Gaotong Medical Technology Company,LTD,China), was placed through the puncture trocar. To reconfirm the ganglion, single stimulation (lasting for $1 \mathrm{~s}$, at 1 intensity) in "coagulation" model was given and induced radiating pain to the maxillary area. Sufentanil $10 \mathrm{ug}$ and propofol $100 \mathrm{mg}$ were intravenoused. One minute after injection of the general anesthetic, the coblation operation was performed using three of intensity for coblation (lasting for $30 \mathrm{~s}$, repeat twice) and six of intensity for coagulation (lasting for $30 \mathrm{~s}$, once). After coblation, the patient reported completed pain relief 0/10 on the VAS scale, and autonomic symptoms disappeared 
without any side effects. During the second morning rounds, patient reported she slept very well last night, which was unachievable after the attack of $\mathrm{HZ}$. The patient was satisfied with the effect on postoperative telephone follow-up performed on day 7, month 1 and month 3, pain VAS score remained on $0 / 10$.

\section{Discussion}

$\mathrm{HZ}$ is characterized by unilateral radicular pain and vesicular eruption caused by the VZV, sensory neurons are most often affected, like trigeminal ganglion, which is the main sensory neuron of the face. VZV infection of the SPG, mainly a parasympathetic ganglion, is extremely rare, and to our knowledge, the present report is the first to record $\mathrm{HZ}$ result in cluster headache. The mechanism of SPG involvement in VZV is still unclear, the maxillary nerve maybe the underlying reason, which carry sensory fibers to the SPG[4], the latent VZV infection in trigeminal ganglion reactived and would extend through maxillary nerve to SPG.

The SPG has traditionally been considered to be involved in the pathophysiology of $\mathrm{CH}[1,5]$, and radiofrequency of the SPG has been described by Salar et al. in 1987[6].Sanders and Zuurmond [7]performed a retrospective analysis of patients with refractory CHs treated by RFA of the SPG. In this study, $60.7 \%$ of the episodic $\mathrm{CH}$ group experienced complete pain relief, and $30 \%$ of the chronic $\mathrm{CH}$ group achieved complete pain relief. A prospective analysis of 37 patients with $\mathrm{CCH}$ who underwent PRF or RFA of the SPG showed the effectiveness of PRF and RFA was similar[8]. lately, Chen Zheng et al. [9]reported the long-term outcomes of CT-guided PRF of SPG showed effective remission in $95.6 \%$ and $64.3 \%$ of patients with refractory episodic and chronic $\mathrm{CH}$, respectively. But Bendersky, D.C et al[4] presented 3 patients failed to achieve adequate pain relief after PRF and subsequently successful CRF of SPG. The patient in this case report had pain remission about 1 month after PRF but then returned to its baseline level, because PRF works as reversible neuromodulation rather than tissue damage[10]. SPG is the biggest parasympathetic ganglion in the head without motor fibers, for those refractory to PRF, neurolysis of the ganglion should be consider, complications like hypoesthesia of the palate or face are usually transient,in this case, patient was free of any observable side effects after coblation of SPG.Coblation is a relatively new technology and has been used in the treatment of NP.It uses radiofrequency energy to create energized plasma that subsequently interact with organic tissue and posses sufficient energy to break molecular bonds causing tissue dissolution at a low temperature (approximately $\left.40^{\circ} \mathrm{C}-70^{\circ} \mathrm{C}\right)[12$, 13],which could reduce the risk of postoperative numbness after radiofrequency for TN.

Facial hematoma because of damage to the maxillary artery or pterygoid plexus is relatively common under fluoroscopy and CT guidance, because of vessels are radio transparent $[5,14,15]$. But under Doppler ultrasound we could identify vessels beside the fossa and keep away from them in real time guidance. Ultrasound guidance has many advantages compare to fluoscopy and CT,and maybe the future direction of pain management.

\section{Conclusion}


In our case report, the first use of coblation has been successfully reducing $\mathrm{CH}$ after VZV infection of SPG. Coblation supplies a novel navigation in $\mathrm{CH}$ treatment but higher level evidences are needed to verify the efficacy of this technique.

\section{References}

[1]. Robbins, M.S., et al., Treatment of Cluster Headache: The American Headache Society EvidenceBased Guidelines. Headache, 2016. 56(7): p. 1093-106.

[2]. Huang, J., Z. Ni and P. Finch, Gasserian Ganglion and Retrobulbar Nerve Block in the Treatment of Ophthalmic Postherpetic Neuralgia: A Case Report. Pain Pract, 2017. 17(7): p. 961-967.

[3]. Li, Y., et al., Comparison of the short-term outcomes after low-temperature plasma radiofrequency ablation (coblation) in the Gasserian ganglion for the treatment of idiopathic trigeminal neuralgia. Journal of pain research, 2019. 12: p. 1235-1242.

[4]. Bendersky, D.C., S.M. Hem and C.G. Yampolsky, Unsuccessful pulsed radiofrequency of the sphenopalatine ganglion in patients with chronic cluster headache and subsequent successful thermocoagulation. Pain Pract, 2015. 15(5): p. E40-5.

[5]. van Kleef, M., et al., Evidence-based interventional pain medicine according to clinical diagnoses. 2. Cluster headache. Pain Pract, 2009. 9(6): p. 435-42.

[6]. Salar, G., et al., Percutaneous thermocoagulation for sphenopalatine ganglion neuralgia. Acta Neurochir (Wien), 1987. 84(1-2): p. 24-8.

[7]. Sanders, M. and W.W. Zuurmond, Efficacy of sphenopalatine ganglion blockade in 66 patients suffering from cluster headache: a 12- to 70-month follow-up evaluation. J Neurosurg, 1997. 87(6): p. 876-80.

[8]. Salgado-Lopez, L., et al., Efficacy of Sphenopalatine Ganglion Radiofrequency in Refractory Chronic Cluster Headache. World Neurosurg, 2019. 122: p. e262-e269.

[9]. Chen, Z., et al., Long-term outcomes of computerized tomography-guided sphenopalatine ganglion-targeted pulsed radiofrequency for refractory cluster headache. Pain Pract, 2019.

[10]. Cahana, A., et al., Pulsed radiofrequency: current clinical and biological literature available. Pain Med, 2006. 7(5): p. 411-23. 
[11]. Li, H., et al., Low-temperature plasma radiofrequency ablation in phantom limb pain: A case report. Brain circulation, 2018. 4(2): p. 62-64.

[12]. Azzazi, A., S. AlMekawi and M. Zein, Lumbar disc nucleoplasty using coblation technology: clinical outcome. J Neurointerv Surg, 2011. 3(3): p. 288-92.

[13]. Trial, C., et al., Coblation technology for surgical wound debridement: principle, experimental data, and technical data. Int J Low Extrem Wounds, 2012. 11(4): p. 286-92.

[14]. Sanders, M. and W.W. Zuurmond, Efficacy of sphenopalatine ganglion blockade in 66 patients suffering from cluster headache: a 12- to 70-month follow-up evaluation. J Neurosurg, 1997. 87(6): p. 876-80.

[15]. Narouze, S.N., Role of sphenopalatine ganglion neuroablation in the management of cluster headache. Curr Pain Headache Rep, 2010. 14(2): p. 160-3.

प्राप्त

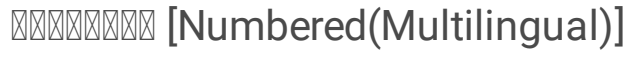

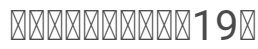

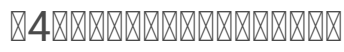

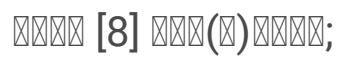

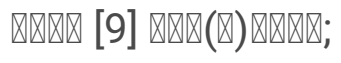

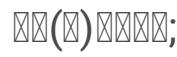

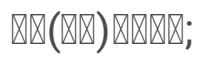

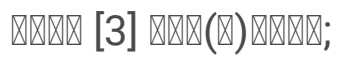

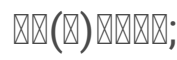

\section{Abbreviations}

CH--Cluster headache; HZ--Herpes zoster; VZV-vesicular-zoster virus; SPG-- sphenopalatine ganglion 


\section{Figures}

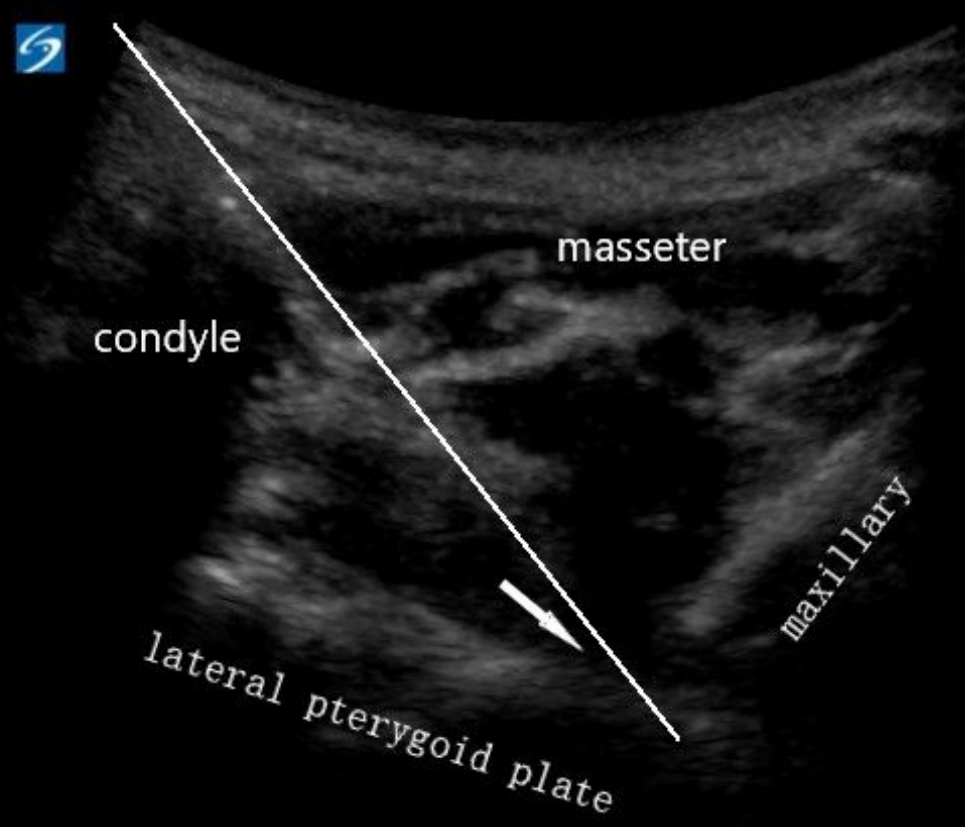

\section{SonoSite}

\section{Figure 2}

Transverse ultrasound image of the pterygopalatine fossa (arrow) and the designed puncture path (line). 
13

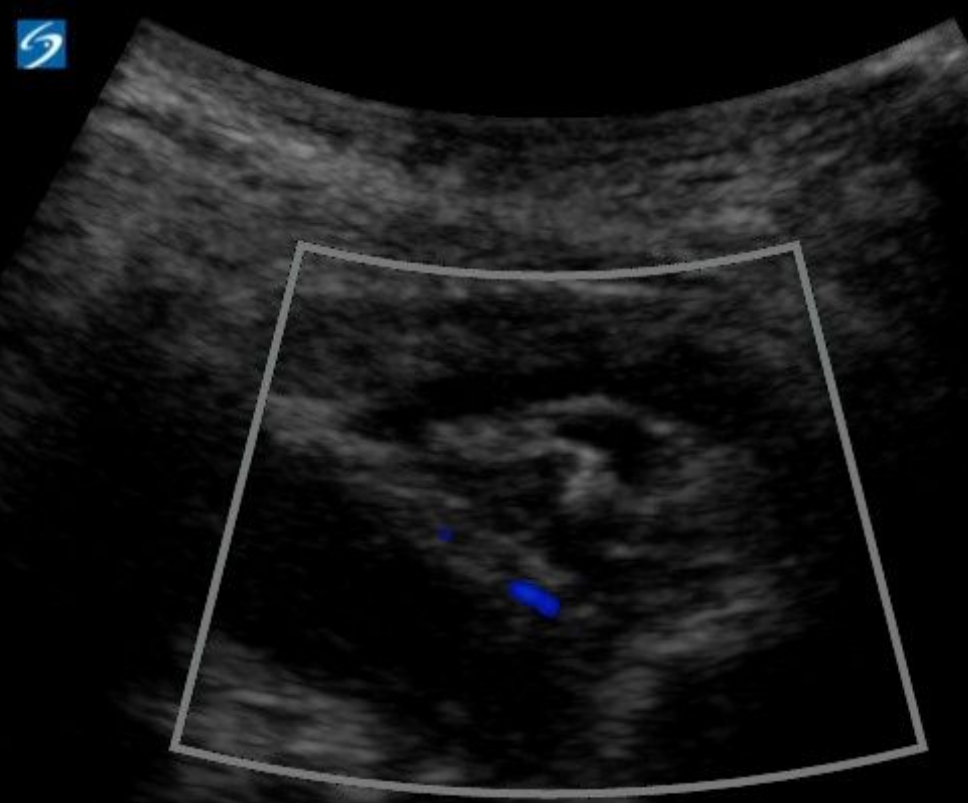

$-13$

$\mathrm{cm} / \mathrm{s}$

\section{SonoSite}

WUBAISHAN

Color: G: 50 2D: G: 50 Low Gen DR: 0 $933 \mathrm{~Hz}$

\section{Figure 4}

Color Doppler shows vessels (blue) besides the pterygopalatine fossa. 


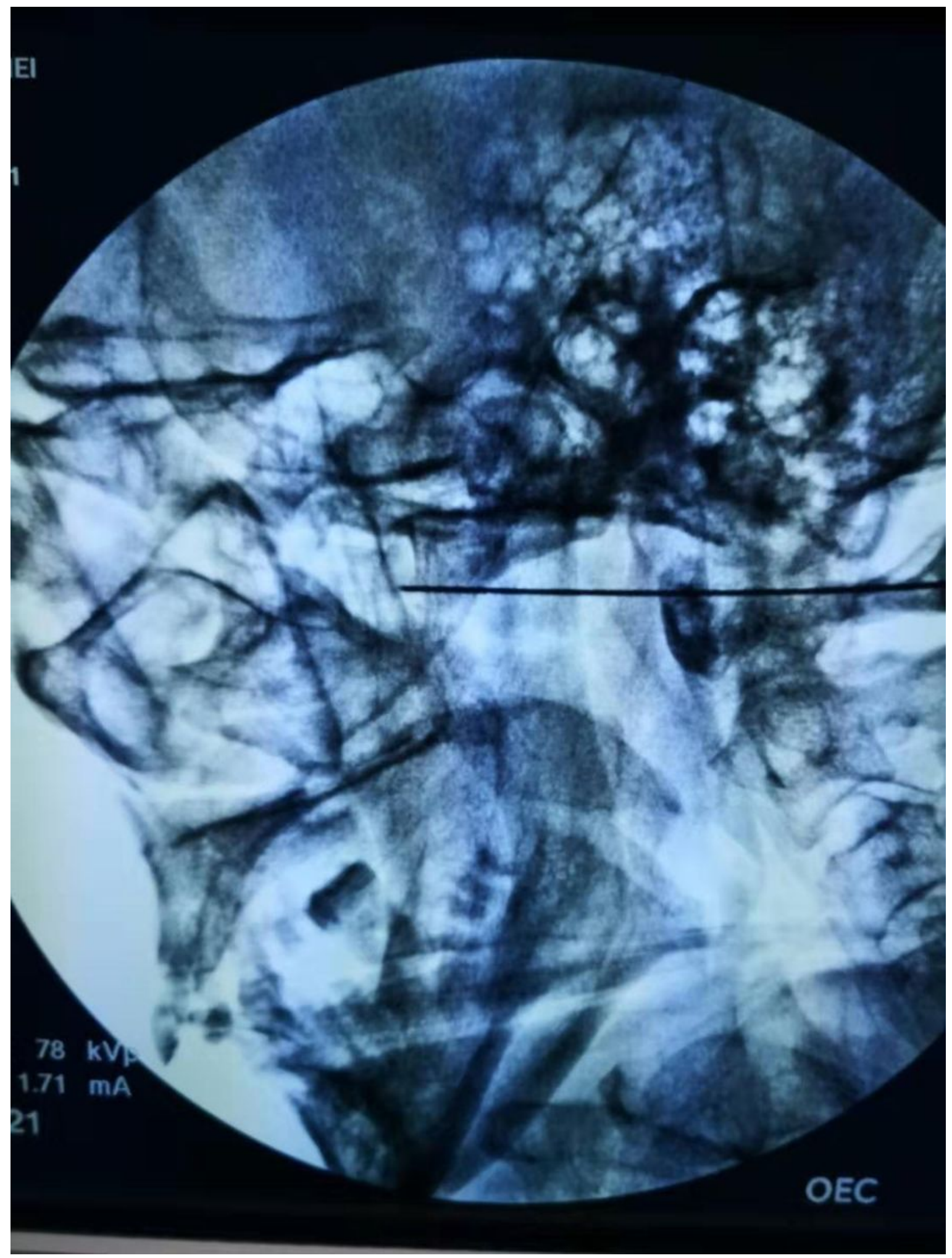

Figure 6

Lateral fluoroscopic image showing the tip of the needle in the pterygopalatine fossa. 


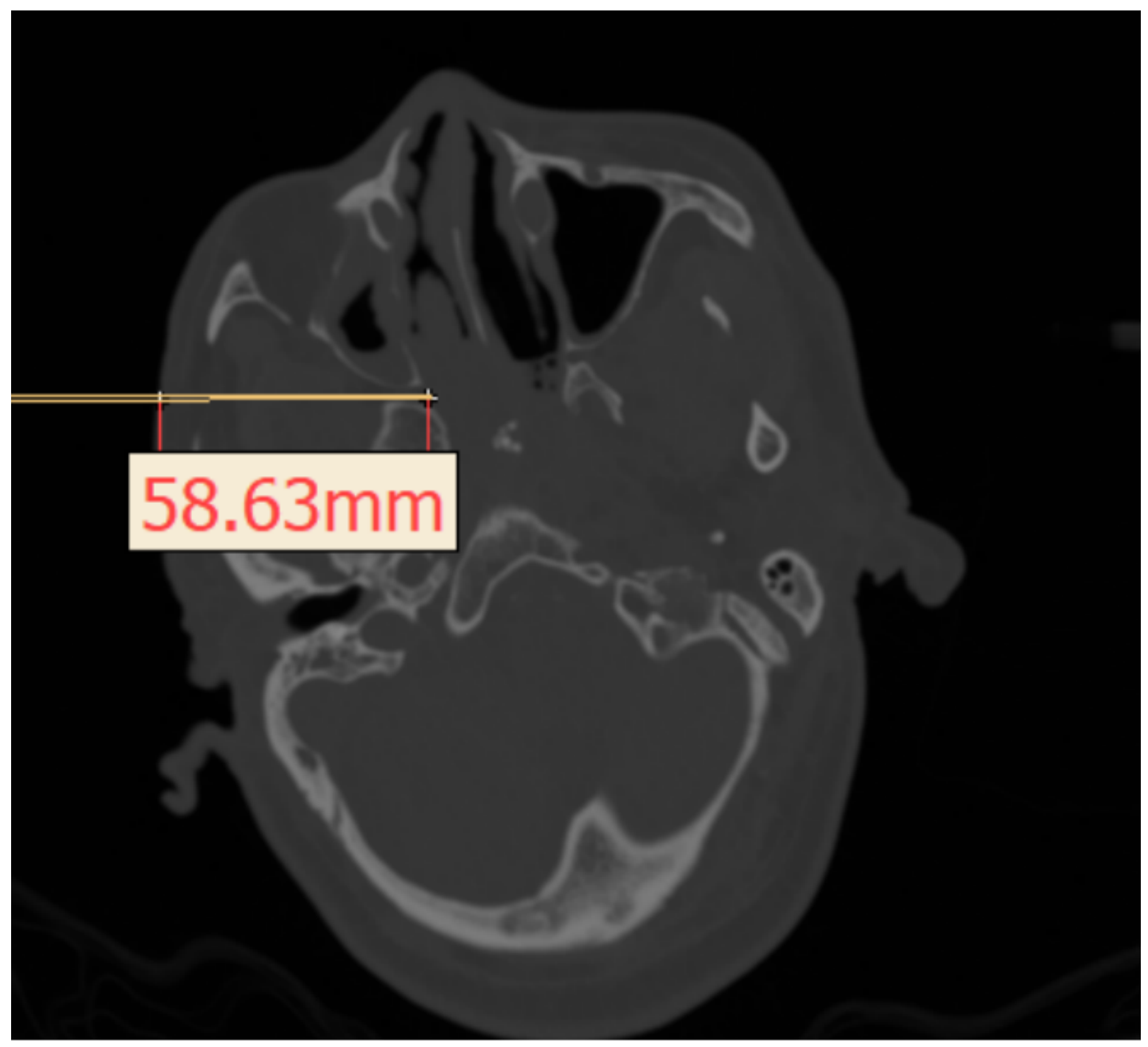

Figure 7

The puncture path was designed used thin-slice CT scans, the depth from skin to pterygopalatine fossa was $58.63 \mathrm{~mm}$.

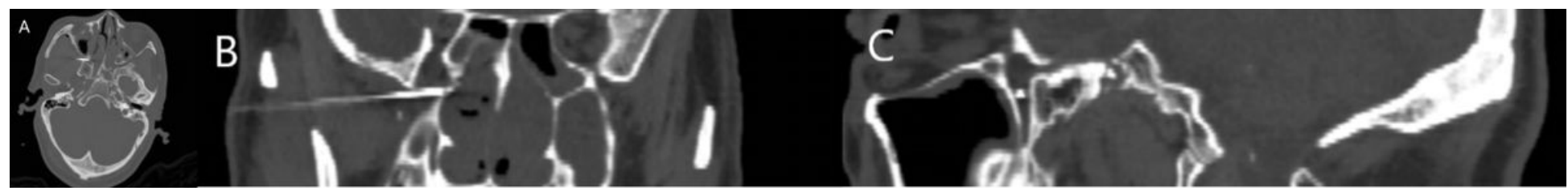

\section{Figure 9}

The Gaotong 17G puncture trocar was inserted to the fossa, and 3D-CT scan to confirm the needle tip 